\title{
5 Ausblick
}

Weitere Untersuchungen der Zelldichten zu früheren und besonders zu späteren Zeitpunkten könnten die Entwicklung der durch die einseitige Elektrostimulation der Cochlea verursachten Effekte verdeutlichen. Untersuchungen an Versuchstieren mit 30 und 140 Tagen Elektrostimulation stehen kurz vor dem Abschluss.

Auch die ABR-Messungen der vorliegenden Arbeit liefern lediglich Daten über den Zeitpunkt der Untersuchung nach 90tägiger Elektrostimulation. Weitere Untersuchungen zu unterschiedlichen Zeitpunkten könnten weiteren Einblick in die Entwicklung der Hörschwellen nach einseitiger Ertaubung und nach einseitiger Elektrostimulation geben. Eine Untersuchung der Hörschwelle nach einem längeren Zeitraum mit einer höheren Anzahl an Versuchstieren könnte z.B. die Frage beantworten, ob neben den beiden gemessenen Hörverlusten der Versuchsgruppe LSR ( 2 von 9 Frequenzen signifikant) weitere entstehen oder ob diese bei einer größeren Stichprobe nicht mehr nachgewiesen werden können.

Die extrazellulären Messungen der vorliegenden Arbeit zeigten im AC nach einer CIStimulation Signale, die im physiologischen Bereich lagen. Mit Verhaltensversuchen könnte zusätzlich bestätigt werden, inwieweit diese Signale nicht nur eine Reaktion im AC auslösen, sondern auch von den Meerschweinchen als verschiedene Tonhöhen wahrgenommen werden.

Eine Untersuchung der SGZ, insbesondere der Zelldichte und des Volumens der Zellen, wäre ebenfalls von großem Interesse. Erwartet wird eine signifikant erhöhte Überlebensrate im elektrostimulierten Ohr, wie in einer Studie (Lousteau, 1987) gezeigt wurde. Die Depolarisation durch die Elektrostimulation scheint das Überleben der SGZ sehr effektiv zu unterstützen (Hansen et al., 2001; Hartshorn et al., 1991; Hegarty et al., 1997).

Weiterhin wäre es interessant zu untersuchen, ob ein Zellverlust einen Einfluss auf die Verarbeitungsqualität der eingehenden Informationen hat oder ob es sich um eine Anpassung des Gehirns auf einen verringerten Informationseingang handelt, der keinen Einfluss auf die Verarbeitungsqualität hat. Untersuchungen zu den Mechanismen der Apoptose/Nekrose, die zum nachgewiesenen Zellverlust führten wären auch sehr von Interesse.

(C) Springer Fachmedien Wiesbaden GmbH 2017

S. Jansen, Chronische intracochleäre Elektrostimulation und ihr

Einfluss auf das auditorische System, DOI 10.1007/978-3-658-18141-3_5 